\title{
Germanica
}

\section{Ernst Jandl : travail langagier et mémoire politique}

Spracharbeit und politisches Gedächntnis.

\section{Elisabeth Kargl}

\section{CpenEdition}

Journals

Édition électronique

URL : http://journals.openedition.org/germanica/529

DOI : 10.4000/germanica.529

ISSN : 2107-0784

Éditeur

Université de Lille

\section{Édition imprimée}

Date de publication : 1 juin 2008

Pagination : 189-208

ISBN : $978-2-913857-21-6$

ISSN : 0984-2632

\section{Référence électronique}

Elisabeth Kargl, «Ernst Jandl : travail langagier et mémoire politique », Germanica [En ligne], 42 | 2008, mis en ligne le 01 juin 2010, consulté le 06 octobre 2020. URL : http://journals.openedition.org/ germanica/529; DOI : https://doi.org/10.4000/germanica.529

Ce document a été généré automatiquement le 6 octobre 2020.

(c) Tous droits réservés 


\title{
Ernst Jandl : travail langagier et mémoire politique
}

Spracharbeit und politisches Gedächntnis.

\author{
Elisabeth Kargl
}

Fünfundvierzig Jahre umfassen Ernst Jandls

Gedichte. Es steckt sozusagen die ganze österreichische Nachkriegsgeschichte im Jandl

drin. $^{1}$

\section{La politique de l'oubli en Autriche}

1 Les années d'après-guerre en Autriche sont marquées par une politique de l'oubli : le premier paragraphe de la «déclaration de Moscou » de $1943^{2}$ stipulant que l'Autriche peut être considérée comme la première victime des nazis, fut le fondement de l'idéologie politique officielle autrichienne après la Deuxième Guerre mondiale ${ }^{3}$. Passer outre le troisième paragraphe de cette déclaration qui stipule aussi clairement que l'Autriche, en participant à la guerre du côté de l'Allemagne nazie, a une responsabilité dont elle ne peut se défaire, s'avère extrêmement commode pour la jeune République qui fonde son identité sur ce déni en s'exonérant ainsi de toute culpabilité. La restauration autrichienne est caractérisée par une aversion massive envers tout ce qui est "allemand", et dans ce sens, les crimes nazis devinrent un "problème " strictement allemand. Il fallait donc mettre entre parenthèses le nazisme et se concentrer plutôt sur la création d'un État autrichien. Cela signifiait concrètement revenir à un héritage culturel d'avant-guerre, et notamment de l'époque de la monarchie. C'est ainsi que films et romans dits de «terroir » («Heimatfilme/-romane»), magnifiant le pays et sa culture, fleurirent - occultant l'existence de l'austrofascisme et $\mathrm{du}$ Troisième Reich. La consolidation de la deuxième République s'établissait seulement sur certains aspects du passé, cette période de restauration marquant clairement une régression et en même temps une mise en scène d'une Autriche mythique présentée comme « île des bienheureux ». 
2 On peut donc difficilement parler d'un point zéro après la Deuxième Guerre mondiale. $\mathrm{Au}$ sein du champ culturel et notamment littéraire, ce consensus de l'oubli est également favorisé par l'intégration rapide des auteurs autrefois pro-nazis comme Max Mell et Heinrich Waggerl, lauréats de prix décernés par l'État. L'extrait du texte Gruß des Dichters, adressé par Alexander Lernet-Holenia, futur président du Pen-Club autrichien, à la rédaction de la revue Der Turm, peut être lu comme le programme de cette époque :

In der Tat brauchen wir nur dort fortzusetzen, wo uns die Träume eines Irren unterbrochen haben, in der Tat brauchen wir nicht voraus, sondern nur zurückblicken. Um es vollkommen klar zu sagen : wir haben es nicht nötig, mit der Zukunft zu kokettieren und nebulose Projekte zu machen, wir sind, im besten und wertvollsten Verstande, unsere Vergangenheit, wir haben uns nur zu besinnen, daß wir unsere Vergangenheit sind - und sie wird unsere Zukunft werden.

3 Le refus de ce consensus se fait moins dans la politique concrète que dans les mouvements intellectuels et notamment parmi les écrivains ${ }^{5}$. Contre le credo de ceux qui prônent "d'oublier et de regarder en arrière", des voix s'élèvent : ne citons que Aufruf zum Mißtrauen deIlse Aichinger, Die Wolfshaut deHansLebert, Unter Mördern und Irren de Ingeborg Bachmann, Der Herr Karl deCarl Merz et Helmut Qualtinger ainsi que les textes de la Wiener Gruppe et de Ernst Jandl. ${ }^{6}$ Le commentaire de Jandl peut être lu comme un contre-programme à Lernet-Holenia et les défenseurs de la restauration :

Damals, wenige Jahre nach Kriegsende, nicht ausschließlich aber doch auch, gegen Faschismus und Krieg zu schreiben, geschah im Sinn der Parole «niemals vergessen!" und war gemeint als ein Beitrag zur Zukunft, nicht bloß als Entschlackungsprozeß. Man schrieb nach vorn, mit dem Blick nach vorn, auch wenn dahinter, im Kopf, die Vergangenheit dröhnte. (AiG, 257) ${ }^{7}$

4 Dans ce climat extrêmement conservateur, la "jeune génération » a du mal à se faire entendre, on refuse l'avant-garde et les textes critiques sont considérés comme "choquants", «provocateurs» ou encore le «reflet d'un pessimisme juvénile» (Zeyringer).

5 Ainsi, la publication des premiers textes expérimentaux de Ernst Jandl, notamment dans la revue «Neue Wege», fait scandale'. Heidi Pataki décrit ainsi l'émoi que l'avantgarde suscite alors en Autriche :

Den gesellschaftlichen Skandal, den die Lautgedichte einst ausgelöst haben, kann sich ein jüngerer Mensch kaum mehr vorstellen. [...] Eine rasende Meute aus Hofräten, Journalisten, Zeitungslesern stürzte sich auf den Lautdichter, auf die Montagenmacher, auf die Wiener Gruppe und den Wiener Aktionismus. Anders in Deutschland. Dort wurden die Avantgardisten eher ironisch belächelt oder ignoriert; sie konnten jedenfalls einen feinen kleinen Elitekreis kultivieren, aber sie wurden keineswegs wütend gehasst ${ }^{10}$.

6 La "nouvelle littérature ", clairement en rupture avec une tradition littéraire conventionnelle, est taxée de "Schmutz-und Schundliteratur », une appellation qui va se maintenir jusqu'aux années 80 et 90 puisque même les textes de Bernhard ou encore de Jelinek se voient qualifiés ainsi.

7 C'est seulement à la fin des années 60 qu'apparaissent des signes d'ouverture avec notamment la création du «Forum Stadtpark» à Graz, le festival d'avant-garde "steirischer herbst », les « rauriser literaturtage » et la publication d'auteurs critiques et novateurs par des maisons d'édition autrichiennes, comme le Residenz Verlag à Salzbourg. 
8 À l'instar de nombreux auteurs des années 50 et 60 , Ernst Jandl a ainsi beaucoup de mal à publier ses textes. Son premier recueil, Andere Augen, poèmes écrits dans la lignée de Brecht et plutôt traditionnels est bien édité en Autriche mais ne connaît pas un grand succès ${ }^{11}$. Jandl se tourne ensuite vers des éditeurs suisses et allemands et c'est ainsi que, en 1966, le Walter Verlag, pourtant plutôt conservateur et catholique, accepte le recueil Laut und Luise. Cette publication provoque néanmoins le renvoi de Otto F. Walter, qui, suivi par de nombreux auteurs, s'en va chez Luchterhand, qui restera par la suite la Maison d'édition de Ernst Jandl.

9 Même si l'on sait que les premiers textes expérimentaux de Jandl ont provoqué un certain émoi, l'écrivain est désormais plutôt connu comme un auteur humoristique maniant avec dextérité la langue et sachant en jouer sur tous les registres. Des textes comme ottos mops ou lichtung sont depuis longtemps étudiés à l'école ${ }^{12}$ et ont certes contribué à une renommée importante de Ernst Jandl, mais ont en même temps fait oublier quelque peu le caractère engagé de cette œuvre en la réduisant à son seul aspect ludique.

Dans ses textes, Jandl s'insurge, partant de son propre vécu ${ }^{13}$, contre la guerre et ses conséquences (comme par exemple dans les poèmes très connus schtzngrmm, vater erzähl vom krieg ou encore der krieg), et aussi, plus généralement, contre l'injustice sociale, la pauvreté, la faim et l'isolement des individus. Plusieurs poèmes sont aussi consacrés à l'implication de l'Autriche dans le national-socialisme et à la politique de l'oubli qui en découla. Jandl est un des premiers à fustiger le passé refoulé de la nouvelle République d'Autriche et à appeler à un devoir de mémoire par l'écriture. Nous aimerions regarder de plus près ces textes traitant du travail de mémoire ${ }^{14}$ et que l'on trouve à toutes les époques de création de Jandl, allant du premier recueil Andere Augen en passant par le célèbre wien : heldenplatz publié dans Laut und Luise, jusqu'à ses textes écrits dans les années 90 , stanzen ${ }^{15}$.

\section{L'œuvre de Ernst Jandl}

11 L'œuvre de Ernst Jandl fait partie d'une littérature de « résistance » (Zeyringer), elle est pour son auteur une « réalisation de liberté » («Realisation von Freiheit» AiG, 44). Dans plusieurs interviews ${ }^{16}$ mais aussi dans les commentaires sur son œuvre réunis dans le recueil Autor in Gesellschaft ${ }^{17}$, Ernst Jandl revient sur la question "politique » de ses textes. L'esthétique, intrinsèque aux textes littéraires, est toujours liée aussi à l'aspect politique. Il ne s'agit pourtant pas de réduire les textes esthétiques à un simple message politique - c'est la radicalité de la forme qui s'affirme en radicalité sociale et donc politique $^{18}$. La critique sociale ne se manifeste qu'à travers le travail sur la langue. La dimension critique n'altère en rien le statut d'œuvre littéraire autonome qui, tout en renvoyant parfois à des événements extérieurs, constitue sa propre réalité langagière et, comme le souligne à juste titre Jandl, ne peut être mesurée qu'à l'aune de celle-ci :

Diese Dichtung ist weder meßbar an einer außersprachlich vorgestellten Welt, noch meßbar an den Verbindlichkeiten einer zur praktischen Mitteilung dienenden Sprache. Sie ist autonom, zu messen nur an ihren eigenen Beispielen. (AiG, 13)

12 Jandl sait comme Brecht (et Jelinek va également le souligner plus tard) que l'art ne peut rien contre la réalité politique et sociale ${ }^{19}$, mais l'auteur peut défier et sensibiliser ses lecteurs, puisque ses poèmes sont toujours écrits pour d'autres («Gedichte sind immer für andere da» AiG, 190). Ses textes vont dans le sens du dévoilement 
(«Aufdecken», comme le dit Heidi Pataki, qui voit dans les textes de Jandl un remède contre la bêtise, le pathos), mais Jandl a toujours refusé l'étiquette "poète visionnaire ». Il n'est pas prophète et ne propose pas de solutions, un pessimisme certain se fait d'ailleurs jour dans ses derniers recueils. Le poète ne peut rien contre la destruction, notamment de la vie (et de sa propre vie), d'où une mise en scène du corps vieillissant, de la maladie, de la mort, notamment dans le recueil der gelbe hund.

Dans «Der Dichter, der uns angeht», Jandl stipule clairement que l'impulsion de ses textes est extérieure : «Dichtung, ausgelöst durch die Zusammenstöße eines Menschen mit der Umwelt.» (AiG, 7), et qu'il s'agit d'une écriture qui refuse de conforter les gens dans leur passivité : «[...] Dichtung, die den Menschen in den Ring stellt, statt ihm den Sport vom Sonntag in die Zeitung zu drucken [...]» (AiG, 7). Ainsi, Jandl s'inscrit dans la lignée des auteurs engagés : «Beunruhigt durch die Möglichkeiten zur Ruhe [...] stiftet der Dichter, der uns angeht, Unruhe dort, wo die einen die Furcht lähmt und die andern ruhig schlafen.» (AiG, 7). Cette écriture prend aussi des allures de défi et ne peut que déranger ceux qui veulent « dormir tranquillement ».

Les textes déploient tout leur potentiel critique seulement s'ils sont écrits dans une forme novatrice ${ }^{20}$. Jandl lui-même distingue parmi ses poèmes différentes formes de textes qui ne constituent pas un système fermé mais figurent plutôt comme des «jalons" ou "balises" («Landmarken zur Orientierung»): «Le poème presque en langage courant, le poème parlé exigeant la voix, le poème sonore sans mots, le poème silencieux visuel. $»^{21}$

Ces textes novateurs ne sont pas en premier lieu la destruction de quelque chose d'existant, aussi conventionnel soit-il, mais contribuent, dans leur esthétique spécifique, à élargir les normes artistiques y compris en revenant à un niveau d'avant la barbarie nazie, d'où le lien avec le dadaïsme et des auteurs comme Arp, Schwitters, Stramm, Stein et Joyce - des influences que Jandl cite volontiers :

Es ging hier nicht um ein Zerstören von etwas, es ging auch nicht primär um einen Protest gegen etwas, sondern es ging darum, die Grenzen der Literatur weiter zu ziehen, als sie während der Hitlerzeit gezogen werden durften, denn damals hat man sehr darauf geachtet, wie das deutsche Wort und wie die deutsche Sprache am besten zur Unterstützung des Nationalsozialismus verwendet werden kann. Es ging um das Wiederentdecken und das Wiedererwecken von etwas, das in der Sprache wie in der Musik oder der Malerei - zu Beginn des Jahrhunderts begonnen hatte. ${ }^{22}$

Jandl ne doute pas de la langue elle-même - puisqu'on est dans la langue et qu'on n'en a pas d'autre pour s'exprimer - mais de son usage :

[...] wo keine Alternative ist, gibt es auch keinen Zweifel. [...] Zur Sprache gibt es keine Alternative, und daher auch keinen 'Zweifel an der Sprache'. Das wird nicht widerlegt durch die Möglichkeit, an eine Vernichtung der Sprache, als Folge einer Vernichtung der Menschheit zu denken; daß diese, als Werk des Menschen, denkbar ist, löst nicht Zweifel an der Sprache aus, sondern Zweifel am Menschen. (AiG, 80)

17 La langue est considérée comme du matériau brut et le poète puise non seulement dans le système de la langue au sens saussurien mais aussi dans son répertoire propre, subjectif: son "réservoir» de mots et de tournures lexicales, de réflexions, de souvenirs etc. Le résultat est la production d'un objet, d'un artefact («das Ergebnis eines solchen Machens», AiG, 60).

Il faut jouer de toutes les possibilités de la langue et Jandl parle de «Manipulation mit dem Sprachmaterial» qui permet de rendre compte de sa composition/construction et 
donc aussi de sa contamination (dans la foulée de Kraus) par des automatismes et des phrases toutes faites. Mais tout comme Jelinek plus tard, Jandl va bien plus loin que Kraus - c'est par la déformation de la langue produisant une nouvelle langue artificielle que l'auteur met à jour le fonctionnement de la langue et de la pensée. La confrontation de matériaux hétérogènes provoque des réactions fortes et le travail sur la lettre («am Wort [sind, E.K.] die größten Veränderungen erzeugbar : Entstellungen, Mißbildungen, andere Wörter. Auswahl, Umformung, Amputation, Transplantation [...]», AiG, 9) permet de rendre visible l'invisible: «Unbekanntes wird am deutlichsten, wenn es neben Bekanntes tritt» (AiG, 9). Cette poésie, même dans son stade le plus expérimental, n'est jamais autoréférentielle ${ }^{23}$, le travail sur le matériau participant toujours du dévoilement de normes et du langage de la société.

Dans de nombreux textes de Jandl, le travail sur la lettre et l'élargissement des formes s'érige contre la politique de l'oubli d'une République volontairement amnésique.

La publication du recueil Andere Augen est plutôt passée inaperçue, comme nous l'avons vu dans le commentaire de Jandl lui-même. Il s'agit ici de textes d'une facture somme toute encore assez conventionnelle dans lesquels le travail sur la langue n'est pas encore très développé. Certains textes, écrits dans la lignée de Brecht, se font critiques de la guerre en général, comme Sich zu erinnern et Der Offiziersbewerber datant tous les deux de 1954. Zehn-Jahre-Pamphlet, écrit une année plus tard, s'élève contre la réintroduction du service militaire en Autriche, Jandl se joignant dans cette critique à celle de H. C. Artmann.

\section{deutsches gedicht}

21 En revanche, nous trouvons dans ce recueil un texte intitulé deutsches gedicht (datant de 1957) qui, à côté de passages plus narratifs et probablement autobiographiques, semble davantage être focalisé sur la forme et le travail sur la langue (notamment en jouant sur une structure musicale spécifique), anticipant ainsi les textes ultérieurs :

ich habe mir um zehn groschen auf der kärntnerstraße ein

hakenkreuz gekauft

ich habe das hakenkreuz auf den rock gesteckt

ich bin in der kärntnerstraße auf und ab gegangen

viele sind in der kärntnerstraße auf und ab gegangen

und haben den arm gehoben und heil gesagt

und ich habe das hakenkreuz auf dem rock gehabt

und heil sagen probiert

und es ist mir im hals steckengeblieben weil es neu war

aber dann ist es ganz gut gegangen

dann bin ich zum zahnarzt gegangen

und ich habe das hakenkreuz auf dem rock gehabt

und die alten frauen im wartezimmer haben mich angeschaut

und ich habe geschaut ob ein magazin auf dem tisch liegt

obwohl ich mir ein magazin nicht gerne anschaue wenn

andere leute da sind

weil in dem magazin nackte frauen sind

sonst würde ich mir das magazin nicht anschauen

aber so etwas sieht man zu hause nicht

außer durchs schlüsselloch die mutter

dann war ich der nächste

und der zahnarzt hat nicht gesagt ich bin ein schwein 
weil mein mund gestunken hat

sondern er hat das hakenkreuz angeschaut und zu

meinem vater gesagt

ich werde noch ein tüchtiger bursch werden (PW 1, 157sq)

Par la description de cet événement dans la vie d'un jeune garçon, Jandl anticipe les thèmes travaillés dans wien: heldenplatz - la complaisance de la foule, l'acceptation sociale du salut hitlérien et d'autres signes ostentatoires comme le port de la croix gammée. Les autorités (ici le médecin) soutiennent et encouragent le prétendu engagement du garçon. Le jeune garçon lie ces événements à des expériences sexuelles comme nous allons également le voir dans heldenplatz. D'autres passages (notamment la description de la foule en extase, le garçon qui s'y trouve coincé, le cri d'une femme, l'aspect de fête populaire, l'imperator qui prend possession de la ville, le motif du chasseur etc.) renvoient par ailleurs au poème publié dans Laut und Luise. Dans deutsches gedicht, nous trouvons aussi des passages plus expérimentaux au niveau de la langue (par des moyens formels comme la répétition, l'inversion, les allitérations, les jeux de mots etc.) qu'on va rencontrer dans des poèmes plus tardifs. Ces passages ne se réfèrent plus à la période de l'Anschluss, mais directement à l'extermination des juifs :

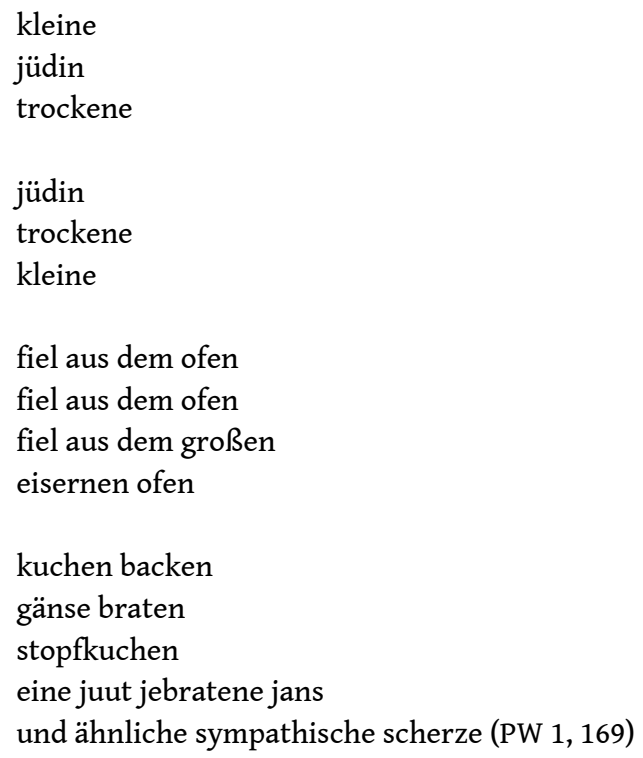

Dans les deux dernières parties du texte, Jandl puise dans un réservoir intertextuel : la structure des strophes fait penser aux comptines ou chansons pour enfants, ce qui est également souligné par le vers «kuchen backen» (faisant allusion à la chanson «backe backe Kuchen»). Jandl détourne le mot «ofen» dont la signification horrible au début de cet extrait de texte est claire, pour être ensuite aussi ramenée à son acception quotidienne, ce qui permet à l'auteur d'énumérer une préparation de plats qui font penser au faste des contes et des fêtes de Noël. «Stopfkuchen» n'est pas une recette proprement dite mais le titre d'un roman de Wilhelm Raabe qui met en scène un personnage du même nom, considéré comme gras et bête dans son enfance. À cela, Jandl ajoute une locution berlinoise connue («Eene jut jebratene Jans is "ne jute Jabe Jottes»). En allongeant encore les voyelles de «juut», il joue sur des effets de prononciation univoques. En prenant les vers des comptines et les éléments des contes au pied de la lettre, ces significations à première vue banales peuvent dévoiler toute leur horreur. Le détournement de connaissances populaires, l'introduction d'éléments 
intertextuels et leur montage avec des événements concrets autour de la préparation de « repas » fait encore mieux ressortir l'atrocité de la Shoah.

Dans «Vorrede zu 'deutsches gedicht'», Jandl note que Rühm et Artmann n'avaient guère apprécié ce texte, et Jandl suppose qu'il était alors tout simplement «trop politique» («das verstieß offenbar gegen Vorstellungen von Reinheit der Kunst. Vorstellungen, die ich selbst nie gehabt hatte.» AiG, 72).

\section{wien : heldenplatz}

Jandl n'a jamais essayé d'être "moins direct » ou de renoncer à la teneur critique/et politique de ses textes. Dans Laut und Luise, le recueil qui a permis à Jandl de se faire connaître, certains poèmes sont regroupés sous le titre krieg und so. Parmi ces textes se trouvent notamment wien : heldenplatz et schtzngrmm, deux textes les plus connus parmi des textes « politiques» de Jandl.

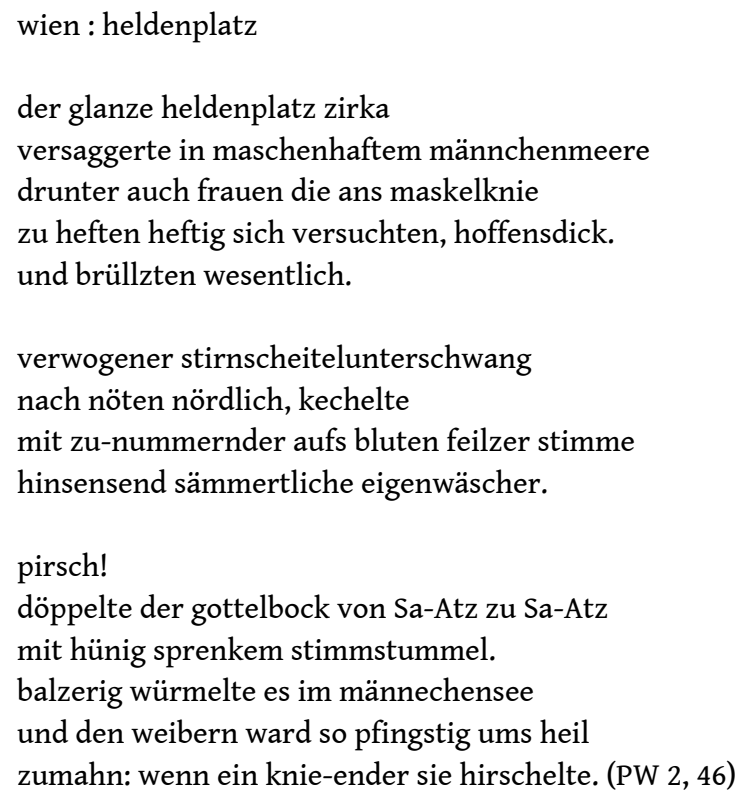

heldenplat $z^{24}$, écrit dans un langage d'une imagination verbale foisonnante, jouant avant tout sur des effets sonores, est un exemple probant de cet appel au travail de mémoire. Nous y trouvons certaines techniques d'écriture que Jelinek va déployer plus tard dans ses œuvres, notamment dans Burgtheater et dans Krankheit oder Moderne Frauen.

Le texte se réfère à une situation historique concrète que Jandl a vécue à 14 ans («l'impulsion extérieure»), à savoir le discours tenu par Hitler à Vienne, sur la Heldenplatz, le 15 mars 1938, et le titre y fait clairement allusion. Jandl explique qu'il se trouvait prisonnier dans une foule très dense et qu'il dut subir les protestations d'une femme qui se sentait importunée par un mouvement du genou du jeune garçon.

Le texte renvoie certes à une réalité historique concrète, mais parvient par des moyens linguistiques sophistiqués à créer une réalité propre, dénonçant le langage des nazis justement par le biais d'une langue défigurée, décriée et persécutée par le régime. Jandl crée une langue artificielle très élaborée, polysémantique, permettant de multiples associations (un peu comme le processus de condensation dans l'analyse des rêves selon Freud ${ }^{25}$ ) sans pour autant détruire la syntaxe, comme le note Jandl lui-même. 
29 Essayons de regarder de plus près le travail sur la langue et les significations et associations qu'il produit. Dès le premier vers, la Place des héros est décrite comme submergée par la foule («der glanze heldenplatz»), mais l'ajout inattendu de la consonne « $1 »$ introduit aussi une nuance de brillance ou de gloire, un effet grotesque d'amplification. Par le «zirka» postposé s'instaure d'emblée une rupture très ironique, faisant allusion au désir consensuel de gommer des faits pourtant bien connus des historiens. Toute la place apparaît ensuite comme noyée, le terme («versaggerte») évoquant à la fois le verbe «versinken» mais aussi «versacken» ou encore «versagen» disparaissant sous une marée humaine («maschenhaftem männchenmeere»). On notera la proximité phonétique entre «Mensch» et «Männchen», qui rappelle bien sûr une masse humaine, mais réduit aussi la population à des animaux, des chiens ou des singes (on songe à l'expression «Männchen machen»), le terme se voulant en tout cas péjoratif. «Maschenhaft» fait aussi bien allusion à «massenhaft» qu'à «Masche», on se perd donc «massivement » dans les mailles du filet national-socialiste, la propagande nazie apparaissant comme une illusion («eine Masche») destinée à appâter les «Männchen».

30 Dans la foule se trouvent aussi des femmes qui essayent à toute force de se tenir («heftig heften») au plus près du «maskelknie», ce dernier évoquant peut-être le «Muskelknie» des autres hommes ou garçons, mais aussi la mascarade en cours. Ces femmes sont «hoffensdick», enceintes («guter Hoffnung»), pleines d'espoir, en attente du grand événement que le Führer promet. La foule, réduite à l'état de créature («das Wesen») «brüllzte wesentlich», l'introduction de «z» renforce la structure consonantique tout en soulignant l'aspect animalier (faisant penser à «lefzen» et «die Lefzen»). Ces effets phonétiques sont importants, on trouve une multitude de "sch", de « $\mathrm{r}$ » ou bien de « $\mathrm{z}$ » (Drews renvoie aussi au « $\mathrm{z}$ laid » chez Arno Schmidt).

$31 \mathrm{Au}$ centre du texte est placé Hitler sans que son nom ne soit cité. Jandl le désigne physiquement et met en scène sa diction particulière, ici transformée en parodie à la Charlie Chaplin : «verwogener stirnscheitelunterschwang » décrit la mèche de cheveux, "verwogen» peut signifier "osé ", mais aussi «fort » comme une vague violente, et le composé renvoie également à «mit Überschwang», avec enthousiasme. Dans l'allitération «nach nöten nördlich», on trouve bien la diction de Hitler qui n'est plus autrichienne mais très allemande, avec des sonorités tournées vers le nord; mais cet énoncé anticipe aussi les «Nöte», les peines à venir. L'orateur est assimilé à un chien : «er kechelte» faisant allusion à «hechelte», littéralement haleter comme un chien, alors que la consonne « $\mathrm{k}$ » renforce à nouveau la sonorité et souligne la part d'agressivité. Hitler vocifère de plus en plus, le volume sonore augmente («zu-nummernder Stimme») évoquant les numéros auxquels les gens se voient réduits; la langue est jusqu'au sang «feilz» très pointue, acérée, l'adjectif se voyant à nouveau renforcé par le « $\mathrm{z}$ » ce qui nous renvoie aux verbes «feilschen», «feilbieten» etc. Le discours est «hinsensend», coupant comme une faux, symbole de la mort, pour tous ceux qui tenteraient de s'y opposer, à savoir «sämmertliche eigenwäscher»; l'adverbe étant une condensation entre «sämtliche» et «jämmerliche», «eigenwäscher» se réfère, selon Jandl, aux individualistes, à ceux qui ne suivaient justement pas la foule.

32 Par son premier vers, le dernier paragraphe évoque clairement les motifs de la chasse qui commence. Ainsi «pirsch !» rappelle soit le nom («die Pirsch»), soit l'injonction par l'impératif. Dans «der gottelbock döppelte», le mâle évoqué est comme Dieu qui saute («hoppeln », doublé justement par « doppeln») de phrase en phrase «von Sa-Atz zu 
Sa-Atz », mais aussi de butin en butin («Atz» signifiant «nourriture» dans la terminologie des chasseurs), tout en évoquant le sigle de la SA. Par "stimmstummel » on peut comprendre des phrases très brèves, saccadées ou marquées par beaucoup d'abréviations (selon Klemperer des signes distinctifs de la LTI, la langue du Troisième Reich), et pourtant la langue est aussi «hünig», faisant appel à «hünenhaft», immense, et «sprenk ", qui peut évoquer le verbe " gesprenkelt », tacheté, mais aussi "streng ", sévère. La foule réagit à cela comme un troupeau d'animaux en chaleur, «balzerig " (en dialecte, on dit bien aussi « gamprig» on " gamsig ", ce qui renvoie à des métaphores animalières), et puis assimilé, à travers un étonnant mot-valise (« es würmelte ») qui associe "Würmer» et « wimmeln», un grouillement d'asticots. Les femmes sont saisies d'émotions ("pfingstig ums heil ») lorsque « ein knie-ender », un homme symbolisé par un cerf avec de nombreuses cornes, les touche («sie hirschelte »). Au contexte de chasse s'ajoutent des connotations clairement sexuelles mais aussi (pseudo-) religieuses. "Heil » évoque bien sûr la tête, mais aussi, dans un contexte de chasse "Weidmannsheil», bonne chasse, et il n'est pas ici utile de mentionner les acceptions du terme sous le régime nazi. Hitler est bien le cerf qui séduit les foules en provoquant des situations d'extase quasi religieuses, comme le décrit aussi Helmut Qualtinger dans Der Herr Karl.

Comme nous pouvons aussi le voir bien plus tard dans les dernières scènes de Krankheit oder Moderne Frauen de Jelinek, la langue a elle-même ici intégré le pathos nationalsocialiste. Elle reflète la destruction, l'agressivité et la pulsion de mort du nazisme. La langue reconstruite renvoie à l'usage que le nazisme a fait de l'allemand, à la corruption de la langue par une barbarie contre laquelle les écrivains doivent s'insurger, en la démasquant par des procédés littéraires novateurs passant notamment par la « contamination » du matériau.

\section{strassenrufe et kurt von schuschnigg}

Dans der künstliche baum et les lese-und sprechgedichte datant de la fin des années 60, nous trouvons plusieurs textes expérimentaux, jouant cette fois encore sur les sonorités et le graphisme - ainsi en est-il d'identität et aussi du poème straßenrufe, dont le titre évoque déjà l'importance de l'oralité:

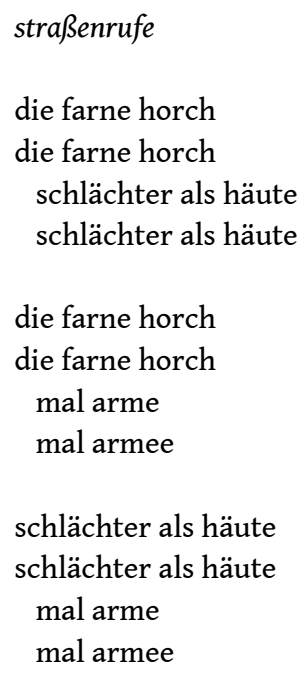


die farne horch

schlächter als heu

schlächter als tee (PW 4, 113)

élément intertextuel : les premiers vers du Horst-Wessel-Lied (« Die Fahne hoch !/Die Reihen fest geschlossen ») et y ajoute l'énoncé «schlechter als heute " qui, dans une première acception, pourrait évoquer une sorte de jugement relevant du politiquement correct ( il faut dire maintenant que la situation d'avant était bien pire que celle d'aujourd'hui »). En jouant sur les effets phoniques et graphiques, ces deux énoncés sont conjugués tout au long du poème permettant de multiples associations mais renvoyant aussi clairement à une "mélodie » guerrière. En jouant sur la proximité phonologique des consonnes, «fahne » devient «farne », bien plus agressif. Le « $\mathrm{r}$ » se trouve également introduit dans l'adjectif, ce qui permet la construction d'un verbe à l'impératif («hoch» devenant «horch»). En lisant le poème à haute voix, et en répétant «farne ", le nom se transforme en musique de "fanfare " qu'on voit défiler dans la rue, soulignant à nouveau le contexte de la « chanson » militaire. Le jeu sur les sonorités est encore plus présent dans le deuxième énoncé, "schlechter » peut être prononcé comme "schlächter » et « heute » devient «häute » dont les connotations sont univoques. «farne » évoque aussi le poème de Stéphane Mallarmé, «L'après-midi d'un faune ", lien mis en scène dans la deuxième strophe. Bien sûr, Jandl utilise le patronyme de Mallarmé pour introduire la signification "arme» tout comme «armee ». Le poème se termine par une étonnante scission de l'adverbe temporel « heute » en introduisant ainsi une chute plutôt inattendue («schlächter als tee »). La critique reste néanmoins très acerbe, voire accentuée par ce point final.

ns un autre poème de la même période, Jandl joue davantage sur les lettres et les effets d'anagrammes. Le texte intitulé kurt von schuschnigg tiré du recueil flöda und der schwan est publié sur deux pages :

kurt von schuschnigg
gott kurze
schütze schürze
österreich fut
(PW 4,172/173)

On peut tout d'abord ne lire que la première page qui semble elle-même univoque. Jandl revient à nouveau sur un événement historique concret, à savoir la dernière phrase $^{26}$ du discours prononcé par le chancelier Kurt Schuschnigg qui, en mars 1938, à l'issue du référendum, remet l'Autriche aux mains de Seyß-Inquart, désigné par les nazis. À nouveau et apparemment sur le mode de la dérision, Jandl introduit une critique très virulente, aussi bien du catholicisme d'État que de l'austrofascisme ouvrant la voie au nazisme. Dans le deuxième texte qui préserve la structure rythmique $\mathrm{du}$ premier tout en déplaçant lettres et syllabes, Jandl parvient à jouer sur de nombreuses connotations différentes. Il combine tout d'abord « kurt » et "schütze », qu'il modifie en «kurze», et sur le mode de l'allitération il transforme «schütze » en "schürze ", n'évoquant pas seulement le tablier mais aussi "Schurz", une sorte de pagne couvrant à peine le sexe évoqué dans le texte. Dans le dernier vers en effet, «kurt» se transforme justement en «fut», perdant ainsi le « $\mathrm{r}$ » utilisé plus haut. «Fut », l'appellation vulgaire du vagin, permet d'établir une connotation nette avec la prostitution - l'Autriche qui se couche pour le régime nazi, ayant été vendue par Schuschnigg, même si celui-ci prétendait tout le contraire. Toute la critique de Jandl se 
trouve dans le travail sur le matériau, sur le va-et-vient entre un agencement particulier des lettres et leur prononciation libérant le sens dans de multiples directions, mais dans un sens politique univoque.

\section{wischen möchten}

Les textes critiques envers l'Autriche et son passé publiés au début des années 70 dans le recueil wischen möchten, déploient encore d'autres moyens littéraires. En jouant sur des effets humoristiques, ils anticipent en quelque sorte les stances des années 90 . Les textes sont précédés de deux épigraphes :

motto 1

auch hitler war ein österreicher

nicht nur christus

motto 2

hermann sei ein fesches haus

komm auf den balkon heraus

(wiener volksspruch, 1938) (PW 6, 161)

La première épigraphe joue sur des évidences, des croyances «établies " de l'État autrichien qui revendique bien la tradition catholique mais qui passe sous silence le fait que Hitler était «également» Autrichien. En instaurant cette contre-vérité ironique, Jandl se moque ici de l'idéologie d'après-guerre lorsque les Autrichiens réussissaient « à faire passer Beethoven comme Autrichien en laissant Hitler aux Allemands ». Dans son commentaire à propos de deutsches gedicht, Jandl précise qu'il a toujours considéré Hitler comme «100\% Autrichien » (PW 1, 173), et, d'une manière très ironique, il prend ici soin de le rappeler à ses compatriotes.

Le prénom Hermann dans la deuxième épigraphe fait probablement allusion à Hermann Göring qui avait été chargé de préparer l'Anschluss. Der «balkon » semble évoquer le fameux balcon qui donne sur la Heldenplatz et d'où Hitler s'adressa le 15 mars 1938 à une foule en liesse, mais peut aussi faire allusion au balcon de l'hôtel de ville de Linz d'où fut proclamé le 12 mars le "Großdeutsches Reich». "fesch» peut d'une part renvoyer au physique et au goût des apparences de Göring, et « ein fesches Haus sein » est une expression viennoise voulant dire " être sympa »; le tout pouvant signifier quelque chose comme "Hermann, toi qui as tout préparé, montre-toi au peuple ». Le fait de signaler qu'il s'agit d'un proverbe populaire («Volksspruch»), met en scène une fausse intertextualité, déconstruite par l'apposition de l'année («1938»).

41 Si les deux épigraphes annoncent clairement la couleur, leur thème et leur ton ironique sont repris dans les textes qui suivent, notamment heimkehr (PW 6, 167) ${ }^{27}$ et le poème sans titre qui lui fait suite :

sie sprechen kriegsverbrecher frei

weil keiner ein ganz schlechter sei

(machtn halt mal mit juden schluß

die man doch nicht vermissen muß)

und alle sagen: österreich

und außerdem: ich komme gleich (PW 6, 185)

Ce texte évoque une fois de plus un contexte concret, à savoir la pseudo-dénazification en Autriche et l'amnistie de 1949 qui s'en suivit. On connaît les jugements très cléments 
et la réintégration rapide d'anciens nazis dans la société et l'appareil d'Etat. Le texte de Jandl anticipe les débats autour de l'élection de Kurt Waldheim à la présidence de l'Autriche en 1986, où avait cours la devise " on n'a fait que notre devoir ». La mise en parenthèses ironique est très explicite : il ne s'agissait pas de vrais crimes puisque cela concernait seulement des juifs qui ne manqueront à personne. Elle évoque donc matériellement par un acte langagier une mise entre parenthèse historique. Les deux derniers vers introduisent une nette rupture renvoyant à la nouvelle République, à l'idéologie du consensus stipulant qu'il faut oublier pour conjuguer tous les efforts, de manière à reconstruire cette République. «Und alle sagen : österreich » ironise cette insistance sur l'austriacité pendant l'immédiat après-guerre, en opposition à tout ce qui est allemand. L'énoncé «ich komme gleich » peut être lu comme un clin d'œil à la deuxième épigraphe, mais peut aussi plus généralement être vu comme une volonté d'adhésion à la jeune République, ce qui permet à Jandl de mettre en cause le suivisme et l'opportunisme incarné par la figure de Herr Karl se déclarant aussitôt fervent démocrate dès la fin de la guerre.

\section{Poèmes des années 90}

43 L'ironie et l'humour noir se retrouvent encore en liaison avec l'intention politique dans les textes publiés dans les années 90, mais les moyens langagiers connaissent de nouvelles mutations. Des vers brefs, caustiques imitant des proverbes populaires, Jandl passe aux stanzen écrites dans un langage artificiel utilisant les ressources du dialecte viennois.

Le fait d'utiliser sciemment le dialecte ou encore de créer un langage artificiel à partir $\mathrm{du}$ dialecte (comme Jelinek va également le faire plus tard dans Burgtheater) met l'accent sur le potentiel subversif du parler populaire s'inscrivant dans une tradition déjà établie dans le théâtre de Nestroy. En général, l'utilisation du dialecte renvoie aux échanges oraux et au parler de classes sociales spécifiques, pas seulement rurales. Le dialecte est facteur d'identité, mais de manière ambiguë. Il est d'une part le parler du peuple contre la langue officielle mais aussi l'expression d'un peuple qui a « failli » (les Viennois de la Place des héros).

Dans le contexte d'après-guerre, l'utilisation du dialecte évoquait souvent l'idylle et était liée à un discours sur la nation, à un patriotisme «local $»^{28}$. Ainsi le dialecte étaitil souvent détourné dans les films dits de terroir où il servait essentiellement à édulcorer (et donc masquer) une réalité sociale et historique. Dans les années cinquante, des auteurs comme H. C. Artmann ou Gerhard Rühm (ne citons que son poème mia san mia) se servent du dialecte comme matériau poétique et démontrent que celui-ci se prête très bien à toutes sortes de manipulation et jeux de langage et ceci justement pour mettre en question la langue manipulée. Le dialecte ou faux dialecte est donc utilisé en opposition à cette littérature du terroir qui glorifie (et fausse) le parler populaire. Le fait d'introduire à l'écrit un registre plutôt parlé, provoque déjà une certaine déstabilisation et déjoue les habitudes. On prend maintenant ce parler au pied de la lettre et on le retourne contre cette fausse culture du peuple, contre l'idylle et le beau, mais aussi contre le beau langage des discours officiels.

Le « dialecte » que l'on trouve dans les textes de Jandl n'a pas de valeur de mimesis d'un dialecte spécifique ${ }^{29}$, mais apparaît plutôt comme langage artificiel très construit. En liaison avec un genre lyrique spécifique lui-même déjà déconstruit - les G'stanzln 
auxquelles Jandl recourt n'ont plus grand-chose en commun avec les stances classiques - l'accent est à nouveau mis sur le travail du matériau langagier. Le fait d'utiliser les G'stanzln signifie une sorte de « réduction » au niveau de la langue et du genre qui met à distance ironique la " haute poésie ", liée à un registre spécifique.

La construction très musicale (quatre vers, césure, accentuation spécifique de syllabes) associée à un parler dialectal, bien qu'artificiel - et dont la prononciation spécifique souligne fortement la musicalité - fait ressortir un caractère anarchique, volontairement vulgaire et surtout faussement innocent. Les G'stanzln, dans le milieu rural, remplissaient bien un rôle de critique sociale. Jandl les ressuscite, ses stances s'attaquent à tout: au banal, aux tabous, aux autorités, à la sexualité, aux abus ${ }^{30}$, et, bien sûr, aussi au passé de l'Autriche. Elles apparaissent ainsi comme moyen ultime de dévoilement, comme un outil poétique de remise en question :

nach 45

des woa ima a sozi

um den brauch maruns ned schean

owa de oamen glaanen jungen nazi

mias ma brodeschian bis zu uns ghean (PW 9, 206)

Dans cette stance, Jandl décrit la situation après 1945 : les socialistes n'ont pas besoin d'aide («brauch maruns ned schean»), contrairement aux pauvres petits nazis («de oamen glaanen jungen nazi») qui doivent être protégés («miass ma brodeschian»). Par là est une fois de plus minimisé le rôle actif et conscient que les individus ont pu jouer dans le nazisme, ce qui est souligné par l'utilisation du diminutif et le parler dialectal lui-même. Ces «jeunes » doivent être réintégrés soit dans le parti, soit dans la société en général («bis zu uns ghean»). Jandl fait non seulement allusion à l'amnistie de 1949 qui a permis à de nombreux nazis d'obtenir leurs droits civiques et de participer ainsi aux élections, ce qui, à l'exception du parti communiste, fut approuvé par tous les partis, mais aussi à la politique de Kreisky, assez clémente envers d'anciens nazis et notamment l'ancien officier SS Friedrich Peter.

Une autre stance thématise aussi clairement le négationnisme :

d'oide antisemitin

waa heit gean a jiidin

dos sogn kennt: schauz mi aun

d'nazi hom uns nix daun (PW 9, 200)

50 À l'instar de Herr Karl de Qualtinger (le passage sur le juif Tennenbaum), Jandl met ici en scène une culpabilité retournée - le fait d'avoir survécu à l'holocauste est utilisé comme alibi par les antisémites et anciens nazis eux-mêmes - puisque les juifs sont revenus, l'holocauste n'a pas pu avoir lieu ou bien « n'était pas si grave que ça ».

51 Bien après 1945 et même après Waldheim, ce travail de mémoire ne semble jamais daté puisqu'il se fait toujours par le travail sur la langue. Esthétiquement parlant, Jandl prépare le chemin à des auteurs comme Thomas Bernhard et, peut-être encore plus, Elfriede Jelinek.

\section{Conclusion}

52 À travers ce choix de textes, nous avons pu apercevoir la constante politique tout au long de l'œuvre de Jandl, qui ne perd jamais de son acuité. Nous pouvons néanmoins constater une évolution de formes et moyens langagiers qui renouvellent sans cesse les 
décentrements à partir desquels s'opère la mise en cause du discours officiel ${ }^{31}$. Ce travail sur la lettre est subversion, destruction, décentrement, déstabilisation, avec, en ligne de mire, un message fort et sans concession.

\section{NOTES}

1. Heidi Pataki: «Ohrfeigen für den guten Geschmack», Wespennest 125 (2001).

2. Rolf Steininger, Michael Gehler (Ed.): Österreich im 20. Jahrhundert. Vom Zweiten Weltkrieg bis zur Gegenwart. Wien, Böhlau, 1997, p. 34.

3. Cf. avant tout Klaus Zeyringer: Österreichische Literatur 1945-1998, Innsbruck, Haymon 1999; et Wendelin Schmidt-Dengler: Bruchlinien, Vorlesungen zur österreichischen Literatur 1945-1990, Salzburg, Residenz Verlag 1995; ainsi que l'interview de Ernst Jandl: «ich sehr lieben den deutschen sprach». Peter Huemer im Gespräch mit Ernst Jandl, Wespennest 125 (2001).

4. Über Österreich zu schreiben ist schwer. Österreichische Schriftsteller über Literatur-Heimat-Politik, Ed. Gerald Leitner, Salzburg, Residenz Verlag, 2000.

5. Peter Huemer constate dans l'interview qu'il mène avec Ernst Jandl: «Der einzige systematische Widerstand, der gegen diesen relativ starken, breiten Konsens des Verschweigens und Vergessens angetreten ist, [kam, E.K] aus dem Bereich der Kunst.» «ich sehr lieben den deutschen sprach» Peter Huemer im Gespräch mit Ernst Jandl, Wespennest 125 (2001); et aussi, du moins en ce qui concerne les années 60: «Was sich in der Bundesrepublik als konkret motivierbare politische Unruhe formierte, das war in Österreich ein ästhetischer Protest gegen das in der Kunst vorhandene restaurative Klima.» Klaus Zeyringer: Österreichische Autorinnen und Autoren. Debuts der letzten zwanzig Jahre, Wien, Köln, Böhlau, 1995, p. 19.

6. Ernst Jandl, d'abord proche de la Wiener Gruppe, ne fut pas vraiment intégré dans ce cercle plutôt fermé. Dans le recueil idyllen, le poème verwandte en témoigne : «der vater der wiener gruppe ist h.c. artmann/ die mutter der wiener gruppe ist gerhard rühm/ die kinder der wiener gruppe sind zahllos/ ich bin der onkel» (PW 9, 8). Cf. aussi Kristina Pfoser-Schewig: «, ,...keine Figur in einem gemeinsamen Spiel'. Ernst Jandl und die ,Wiener Gruppe' ", in Die Wiener Gruppe (Walter-Buchebner-Literaturprojekt), Wien, Böhlau, 1987, p. 69-83.

7. Ernst Jandl: Autor in Gesellschaft. Aufsätze und Reden, München, Luchterhand 1999 (AiG).

8. «Gegen die heilige Allianz von ehemaligen Nazis, Austrofaschisten und einigen zurückgekehrten Emigranten - letztere Garanten einer antifaschistischen Einstellung -, die als PEN-Club das literarische Leben des Landes beherrschte, kam die jüngere, die ,zweite' Generation nicht an.» Klaus Zeyringer: Österreichische Autorinnen und Autoren, op. cit., p. 13.

9. Cf. Kristina Pfoser-Schewig: «Die literarischen Arbeiten Ernst Jandls in den 50er Jahren», in Etudes germaniques avril-juin (1995), p. 209-221.

10. Heidi Pataki: «Ohrfeigen für den guten Geschmack», Wespennest 125 (2001).

11. Jandl commente cette première publication dans «Wie kommt man zu einem Verlag?» : «Mein erstes Buch, 1956 in Österreich verlegt, war nie richtig aufgetaucht, doch gründlich untergegangen.» (AiG, 194). Par ailleurs, il ne faut pas oublier que, dans le cadre d'un anticommunisme virulent, Brecht fut boycotté sur les scènes autrichiennes entre 1952 et 1963.

12. Le choix des textes de Jandl pour les livres scolaires reste très restreint et englobe presque exclusivement des textes tirés de Laut und Luise. Cette réception spécifique a contribué à la renommée de Jandl comme «clown des jeux de mots» («Sprachclown und Sprachspiel-Jandl»). 
Korte cité dans Barbara Mühlberger: «Jandl als Lehrer und Dichter. Eine Schulstunde», in Bernhard Fetz (Ed.): Ernst Jandl. Musik Rhythmus Radikale Dichtung, Wien, Paul Zsolnay, 2005, p. 101-103, cit. p. 101.

13. Voir aussi Ernst Jandl: Briefe aus dem Krieg 1943-1946, München, Luchterhand, 2005.

14. À notre connaissance, cet aspect qui pourrait paraître évident aux lecteurs connaissant Jandl, n'a jamais fait l'objet d'un article en France. Nous aimerions néanmoins attirer l'attention sur le travail de Bernard Banoun abordant la question du politique dans la poésie de Ernst Jandl. Bernard Banoun: "Éclats de dire. La politique du poétique chez Jandl le Sérieux ", in La poésie comme geste politique. Tumultes 19 (2002), p. 67- 89.

15. Nous nous référons à l'édition complète des œuvres: Ernst Jandl, Poetische Werke, tome 1-10, München, Luchterhand, 1997. (PW)

16. Notamment dans «ich sehr lieben den deutschen sprach ». Peter Huemer im Gespräch mit Ernst Jandl, Wespennest 125 (2001).

17. Ernst Jandl: Autor in Gesellschaft. Aufsätze und Reden, München, Luchterhand 1999 (AiG). Ce recueil reprend aussi les conférences de Francfort (Frankfurter Poetikvorlesungen), publiées d'abord dans Das Öffnen und Schließen des Mundes, Darmstadt, Luchterhand, 1985.

18. «Krieg, das wissen wir, ist ein herausforderndes Thema, aber es läßt sich zu diesem Thema ein herausforderndes Gedicht nur schreiben, wenn das Gedicht sich nicht auf das Herausfordernde seines Themas verläßt, sondern als Gedicht selbst, ungeachtet des Themas, zu einer Herausforderung wird. Das kann nur durch Veränderungen am Gedicht, seiner Sprache und seiner Struktur geschehen, Veränderungen, die im Gegensatz zu allem stehen, woran wir uns gewöhnt haben.» (AiG, 191).

19. Ernst Jandl se situe aussi concrètement du côté de la protestation : nous ne mentionnerons ici que son engagement contre le très conservateur PEN-Club (qui s'insurgea contre la remise du prix Nobel à Heinrich Böll) et pour la création de la GAV (Grazer Autoren Versammlung) dont Jandl fut le président pendant plusieurs années. Il s'engageait à côté des sociaux-démocrates, ce qui ne l'empêchait pas de les critiquer.

20. «Der schärfste Verstand, die beste Gesinnung, die schlagendste Kritik treffen ins Leere, wenn sie mit abgelutschten Worten und in altmodischer Technik daherlatschen [...] Der wahre politische Dichter muss mit frischer, unverbrauchter, avancierter Technik parieren. Der Überraschungs-Coup muss sitzen! ». Heidi Pataki: "Ohrfeigen für den guten Geschmack », Wespennest 125 (2001).

21. "Das Gedicht in nahezu Alltagssprache; das Stimme verlangende Sprechgedicht; das laute wortlose Lautgedicht; das stille visuelle Gedicht » (AiG, 134). Cf. Bernard Banoun: "Éclats de dire. La politique du poétique chez Jandl le Sérieux», Tumultes 19 (2002), traduction de Bernard Banoun.

22. «ich sehr lieben den deutschen sprach». Peter Huemer im Gespräch mit Ernst Jandl, Wespennest 125 (2001).

23. Cf. Bernard Banoun: « Éclats de dire. La politique du poétique chez Jandl le Sérieux », Tumultes 19 (2002).

24. Comme ce texte a déjà fait l'objet de plusieurs commentaires, nous nous contentons de revenir sur les points essentiels du travail langagier en suivant les remarques de Jandl lui-même («Mein Gedicht und sein Autor», AiG, 34sq) et ceux de certains critiques : Walter Ruprechter: Wien : heldenplatz, in Interpretationen. Gedichte von Ernst Jandl, Stuttgart, Reclam, 2002, p. 34-47; Jörg Drews: «Über ein Gedicht von Ernst Jandl», manuskripte 69/70 (1980), p. 162-164. )

25. Voir Ruprechter: wien : heldenplatz, op. cit., p. 39.

26. «So verabschiede ich mich in dieser Stunde von dem österreichischen Volke mit einem deutschen Wort und einem Herzenswunsch : Gott schütze Österreich. » Friedrich Weissensteiner: Der ungeliebte Staat. Österreich zwischen 1918 und 1938, Wien, ÖBV, 1990, p. 294. 
27. D'autres textes publiés fin 70 dans le recueil der gelbe hund reprennent le même sujet mais sont écrits sur un ton bien plus grave et moins ironique, comme vom aufrechten gang (PW 8, 30) ou nach altem brauch (PW 8,35).

28. Voir plus en détail Klaus Zeyringer: Österreichische Literatur, op. cit., p. 314sq.

29. Cf. Wendelin Schmidt-Dengler: ",noch ein weilchen dichterlich' Zu Ernst Jandls Lyrik von 1982 bis 1992 », Text + Kritik 129 (1996), p. 51-60.

30. Une des stances anticipe notamment l'affaire autour du cardinal Groer en 1995, accusé d'avoir abusé de jeunes garçons, ce qui a provoqué un référendum pour un renouveau au sein de l'Église catholique: "ge fiachd di ned, biawal/bin jo beinoh da bopst/schdecksd dei zipferl in mein mund rein/sogst ma daun, obsdas mogsd» (PW 9, 213).

31. «Jandl hat nicht seine Haltung gewechselt, sondern seine ästhetischen Mittel.» Heidi Pataki: «Ohrfeigen für den guten Geschmack», Wespennest 125 (2001).

\section{RÉSUMÉS}

Ernst Jandl est avant tout connu comme poète drôle, maniant avec dextérité la langue, mais on oublie souvent la constante politique de son oeuvre. Dans de nombreux textes, l'auteur s'insurge contre la guerre et ses conséquences et aussi, plus généralement, contre l'injustice sociale, la pauvreté, la faim et l'isolement des individus. Nous proposons de regarder de plus près un choix de textes - allant du premier recueil Andere Augen en passant par le célèbre wien: heldenplatz jusqu'aux stanzen des années 90 - consacré à l'implication de l'Autriche dans le nationalsocialisme et à la politique de l'oubli qui en découla. Jandl est un des premiers à fustiger le passé refoulé de la nouvelle République d'Autriche et à appeler à un devoir de mémoire par un travail sur la lettre qui est toujours subversion, destruction, décentrement, déstabilisation, avec, en ligne de mire, un message fort et sans concession.

Ernst Jandl ist bekannt als Autor, der das Sprachspiel meisterhaft beherrscht. Dabei wird der engagierte und politische Aspekt seines Werkes oft in den Hintergrund gedrängt. In zahlreichen Texten jedoch klagt der Autor den Krieg und dessen Konsequenzen, aber auch soziale Ungerechtigkeit, Hunger und Einsamkeit der Individuen an. Anhand verschiedener Texte (u.a. aus dem ersten Gedichtband AndereAugen, aber auch wien: heldenplatz und stanzen) soll in diesem Artikel gezeigt werden, wie Jandl mit verschiedenen sprachlichen Mitteln die österreichische nationalsozialistische Vergangenheit und die darauf folgende Verdrängungspolitik thematisiert. Jandl war einer der ersten Autoren, der die verdrängte Vergangenheit der Zweiten Republik anklagte und zur „Aufarbeitung“ dieser Vergangenheit durch Arbeit an der Sprache aufrief. Seine Spracharbeit ist Subversion, Zerstörung, Dezentrierung, Destabilisierung, der eine klare politische Aussage zugrunde liegt.

\section{AUTEURS}

\section{ELISABETH KARGL}

Université de Nantes 